\title{
Scalable Algorithms for Missing Value Imputation
}

\author{
Marghny H. Mohamed \\ Faculty of Computers and Information, \\ Assiut University, Assiut, Egypt
}

\author{
Abdel-Rahiem A. Hashem \\ Mathematics Department, Assiut \\ University, Assiut, Egypt
}

\author{
Mohammed M. Abdelsamea \\ IMT Institute for Advanced Studies, \\ Lucca, Italy
}

\begin{abstract}
Statistical Imputation Techniques have been proposed mainly with the aim of predicting the missing values in the incomplete sets as an essential step in any data analysis framework. K-means-based Imputation, as a representative statistical imputation method, has been producing satisfied results in terms of effectiveness and efficiency in handling popular and freely available data set (e.g., Bupa, Breast Cancer, Pima, etc.). The main idea of K-means based methods is to impute the missing value relying on the prototypes of the representative class and the similarity of the data. However, such kinds of methods share the same limitations of the Kmeans as data mining technique. In this paper and motivated by such drawbacks, we introduce simple and efficient imputation methods based on K-means to deal with the missing data from various classes of data sets. Our proposed methods give higher accuracy than the one given by the standard K-means.
\end{abstract}

\section{General Terms}

Data Mining, Algorithms

\section{Keywords}

Statistical Imputation, Clustering, K-mean

\section{INTRODUCTION}

The quality of mining a data set in any data analysis framework is affected by how complete the data is. As a consequence, the quality of the data attracts the attention of many scientists working on Data mining and other correlated area such as Machine learning. The presence of missing data presents a challenge in the cleaning step, which is occurred in the phase of data collection [1, 2]. As pointed out in [3, 4, 5], we can classify the missing data into three categories: Missing completely at random (MCAR), Missing at random (MAR), and Not missing at random (NMAR).

In the MCAR, the absence of an item is not associated with any other item in the data set, observed or missing. In other words, the distribution of an example when containing a missing value for an attribute does not depend on either the observed data or the missing data. On the other hand, MAR has a less restrictive assumption than MCAR. It indicates that the absence of an item depends only on the observed values in the data set (e.g., the dependency is only for the observed data). Compare to MAR, NMAR produces the opposite condition, which the absence of an item reflects its probable data value [4].

In order to deal with such issues, several treatment missing data methods have been proposed, they can be divided into three categories: First, Ignoring and discarding (ID) category. There are two main ways to discard data with missing values. The first is discarding all instances with missing data while the second is discarding instances and/or attributes. This method relies on the definition and the specification of the high levels of missing data to evaluate its relevance to the analysis. However, the most relevant attributes should be kept even with high degree of missing values. The second category is Parameter Estimation (PE) class. In this class, Maximum likelihood procedures are used to estimate the parameters of a model defined for the complete data (e.g., ExpectationMaximization [6] algorithm is applied in [2] to handle parameter estimation in the presence of missing data). The last category is Imputation $[7,8,9]$, which is proposed with the aim of filling the missing values with estimated ones. The methods presented in this paper focus mainly on the last category.

\section{STATISTICAL IMPUTATION METHODS}

Statistical Imputation is the process of replacing missing values with estimated ones based on some statistical information available in the data set. There are many options varying from naive methods like mean or mode imputation [10] to some more robust methods based on relationships among attributes. Also, Imputation type is determined by how many values to be predicted for the missing one (e.g., single/multiple imputation [11]). In this section, we briefly describe different kind of imputation methods and highlight their limitations.

Mean and mode imputation (Mimpute) [12, 13, 14] consists of replacing the unknown/missing value for a given attribute by the mean (quantitative attribute) or mode (qualitative attribute) of all known/available values of that attribute. However, replacing all missing records with a single value distorts the input data distribution. Hot deck imputation (HDimpute) [15] replaces the missing data with the values from the input vector that is closest in terms of the attributes that are known in both patterns. Unlike Mimpute, this method attempts to preserve the distribution by substituting different observed values for each missing item [12]. Another solution is provided by Cold Deck imputation (CDimpute) method, which is similar to hot deck but the data source must be other than the current data set. On the other hand, Prediction models $[11,14]$ consist of creating a predictive model in order to estimate values that will substitute the missing data. The main idea of the predictive model is to rely on the correlations presented among the attributes to create a predictive model for classification or regression. However, its main disadvantage is that a huge number of prediction models have to be designed when missing items appear in many combinations of attributes in a high dimensional problem. 


\subsection{K-Means based Imputation}

In this section, we review the main idea of the K-means based imputation methods. Once the clusters are constructed, the imputation can be done by the corresponding prototypes from the most similar k-centroid of the given classes. The Classic Imputation algorithm (CI) can be described as follows:

1. Divide dataset $\mathrm{S}$ into Complete-valued dataset $\mathrm{S}^{\mathrm{t}}$, and Missingvalued dataset $S^{*}$.

2. Apply classical k-mean on complete dataset $S^{t}$ until convergence and obtain $w_{\mathbf{j}}$ centers, $\mathbf{j} \in\{1,2, \ldots, \mathrm{k}\}$.

3. For each instance $x_{\mathbf{i}}$ containing missing value, where $x_{\mathbf{i}} \in$ $\mathrm{S}^{*}$. Compute distance between centroid $\mathrm{C}_{\mathbf{j}}$ and instance $\mathrm{x}_{\mathbf{i}}$ containing missing value.

4. Impute missing-value in $\mathbf{x}_{\mathbf{i}}$ from its corresponding closest centroid wj.

In this paper, we enhance an imputation method based on kmeans in several ways by enhancing the way of imputation and giving an efficient accuracy compared with an imputation method based on k-means, which proved to be successful in missing value imputation than other statistical approaches.

\section{PROPSED MODIFICATION OF CLASSIC IMPUTATION METHOD}

When the missing values in the selected sample are exceeding the number of the available ones, this implies that the measured distance will be in (n-p) space, which means inefficient measured distance. Hence, we will improve the missing values imputation by modifying the steps to obtain the measured distance. When we get the first centroids from the clustering process, we initialize missing values by imputing from prototypes of these centroids. So, the distance measure in the next step becomes in $\mathrm{n}$ dimension and, in each new clustering process, imputation will be achieved by measuring the closest distance between whole sample and new centroids. The Modification of Classic Imputation algorithm (MCI) is described as follows: (see Figure 1)

1. Divide data set $\mathrm{S}$ into Complete-valued data set $\mathrm{S}^{\mathrm{t}}$, and Missingvalued data set $S^{*}$.

2. Start K-means algorithm on $\mathrm{S}^{\mathrm{t}}$, while clusters optimized, for each computed centroid $w_{\mathbf{j}}, \mathbf{j} \in\{1,2, \ldots, \mathrm{k}\}$ and missingvalue instance $x_{\mathbf{i}}$, where $\mathbf{x}_{\mathbf{i}} \in \mathrm{S}^{*}$. Compute distance between centroid $\mathrm{w}_{\mathbf{j}}$ and missing-value instance $\mathrm{x}_{\mathbf{i}}$.

3. Impute missing-value in $\mathbf{x}_{\mathbf{i}}$ from its corresponding closest centroid $w_{\mathbf{j}}$.

4. Repeat step 2 and 3 until k-means convergence.

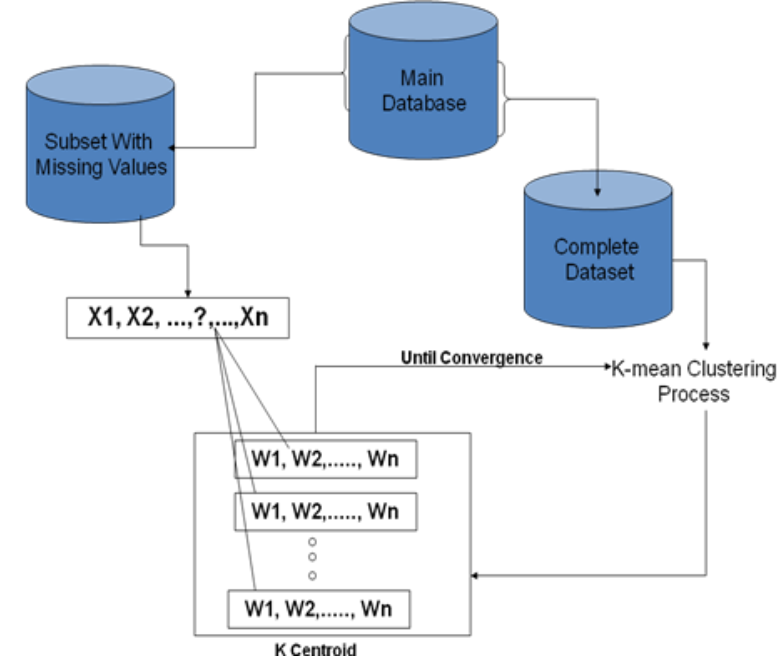

Fig 1: A Modification of classic Imputation based kmeans.

\section{ENHANCEMENT MODIFICATION OF CLASSIC IMPUTATION METHOD}

In each clustering process each sample gets imputed from the centroid of its closest cluster, we count the number of times the sample has been imputed from a particular cluster. The largest number of times a sample gets assigned to a particular cluster means that it belongs to this cluster, which will result in the imputation of the values of the last cluster's centroid of the most visited cluster to the sample. Enhancement of Modification of Classic Imputation algorithm (EMCI) is described as follow:

1. Divide data set $\mathrm{S}$ into Complete-valued data set $\mathrm{S}^{\mathrm{t}}$, and

Missing-valued data set $\mathrm{S}^{*}$.

2. Initialize class counter $\mathrm{CC}_{\mathbf{j}}$ for each missing-value instance, where $\mathbf{j} \in\{1,2, \ldots, \mathrm{k}\}$.

3. Start K-means algorithm on $\mathrm{S}^{\mathrm{t}}$, while clusters optimized, for each computed centroid $w_{\mathbf{j}}, \mathbf{j} \in\{1,2, \ldots, \mathrm{k}\}$ and missing-value instance $\mathbf{x}_{\mathbf{i}}$, where $\mathbf{x}_{\mathbf{i}} \in S^{*}$. Compute distance between centroid $\mathrm{w}_{\mathbf{j}}$ and missing-value instance $\mathbf{x}_{\mathbf{i}}$.

4. Impute missing-value in $\mathrm{x}_{\mathbf{i}}$ from its corresponding closest centroid $w_{\mathbf{j}}$ and increment its corresponding closest center $\mathrm{cc}_{\mathbf{j}}$.

5. Repeat step 3 and 4 until k-means convergence. For each missing-value instance $x_{\mathbf{i}}$, where $\mathrm{x}_{\mathbf{i}} \in \mathrm{S}^{*}$, Choose the maximum class counter and impute missing-value in $\mathbf{x}_{\mathbf{i}}$ with it is corresponding prototype centroid.

\section{EXPERIMENTAL RESULTS}

We choose four real-world data sets from the UCI Machine Learning Repository [3] and compare the three missing value strategies discussed earlier. These data sets are chosen because they have at least some discrete attributes, multi class, and a good number of examples. Then, we will select values from original data sets to be missing to simulate different situations with missing values. To simulate missing values in data sets, we randomly select certain percentages $(2$ percent, 4 percent, 6 percent, 10 percent, 20 percent and 40 percent) of attribute values in the whole data set to be missing and those missing values are distributed into each attribute 
proportional to its cost as more expensive attributes usually have more missing values.

Table 1. Data Sets Used in the Experiments.

\begin{tabular}{|l|c|c|}
\hline Data Base & No. of attributes & No. of examples \\
\hline Iris & 4 & 150 \\
\hline Ecoli & 7 & 336 \\
\hline Bupa & 6 & 345 \\
\hline Pima Indian & 8 & 768 \\
\hline
\end{tabular}

This study shows the performance of three imputation methods based on k-means; Classic Imputation (CI), Modification of Classic Imputation (MCI) and Enhancement of Modification of Classic Imputation (EMCI). Each graph compares the performance of all methods with different level of missing values for different clusters of K-Mean. For the purpose of accuracy, we use the mean square errors which gives from error $=(R-I) 2 / N$ where $R$ is real value, $I$ is Imputed value and $\mathrm{N}$ is number of missing values.

In our experimental results, all figures illustrate the mean square error comparison for the three imputation method describes in previous sections, while all tables illustrate the sum of square errors comparison for simplicity of showing the difference between three methods.

Table 2 illustrates an error comparison between an imputation methods based on k-means, CI, MCI and EMCI in different missing instance percentage at several cluster number for Bupa dataset.

Table 2. A sum of square error comparison of three imputation methods in the Bupa data set.

\begin{tabular}{|c|c|c|c|}
\hline Miss.(\%) & Cluster No & $\begin{array}{c}\text { Imputation } \\
\text { CI }\end{array}$ & $\begin{array}{l}\text { approaches based K-mean } \\
\text { MCI EMCI }\end{array}$ \\
\hline \multirow{4}{*}{2} & 3 & 4.777186 & 3.1867590 .299139 \\
\hline & 4 & 3.581818 & 2.7919750 .309811 \\
\hline & 5 & 0.907024 & 0.9070240 .684761 \\
\hline & 6 & 1.116783 & 1.1167831 .116783 \\
\hline \multirow{4}{*}{4} & 3 & 4.383957 & 3.5886740 .609776 \\
\hline & 4 & 4.39595 & 2.9539050 .489464 \\
\hline & 5 & 1.481603 & 1.4816041 .183803 \\
\hline & 6 & 1.669874 & 1.6698741 .669874 \\
\hline \multirow{4}{*}{6} & 3 & 7.765477 & $6.71576 \quad 4.331328$ \\
\hline & 4 & 6.907715 & 5.6382643 .821443 \\
\hline & 5 & 3.641498 & 3.6414983 .763348 \\
\hline & 6 & 7.375067 & 7.3750677 .375067 \\
\hline \multirow{4}{*}{10} & 3 & 12.01079 & 11.0911810 .11517 \\
\hline & 4 & 11.92479 & 10.600738 .489751 \\
\hline & 5 & 8.332617 & $8.33262 \quad 8.420806$ \\
\hline & 6 & 14.09595 & 14.0959514 .09595 \\
\hline \multirow{4}{*}{20} & 3 & 12.76048 & 11.136196 .747862 \\
\hline & 4 & 11.67825 & 10.884455 .91155 \\
\hline & 5 & 10.84042 & 10.8404110 .16937 \\
\hline & 6 & 23.74567 & 23.7456523 .74565 \\
\hline \multirow{4}{*}{40} & 3 & 18.34416 & 16.7377715 .22232 \\
\hline & 4 & 21.26251 & 20.4683713 .66378 \\
\hline & 5 & 22.84709 & 22.8470719 .59215 \\
\hline & 6 & 47.41088 & 47.4108147 .41081 \\
\hline
\end{tabular}

From table 2 we can notice that the difference between EMCI algorithm and other two methods is clear strongly for little clusters in this data set, Bupa, See figure 2.
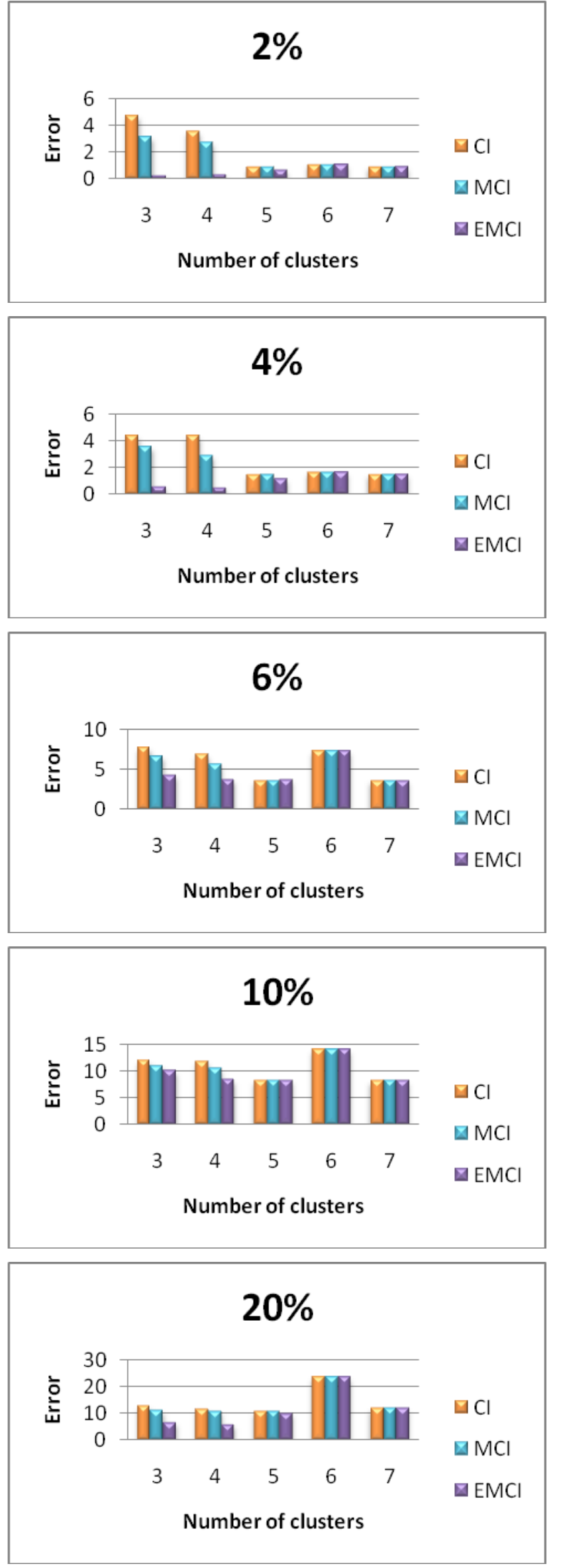


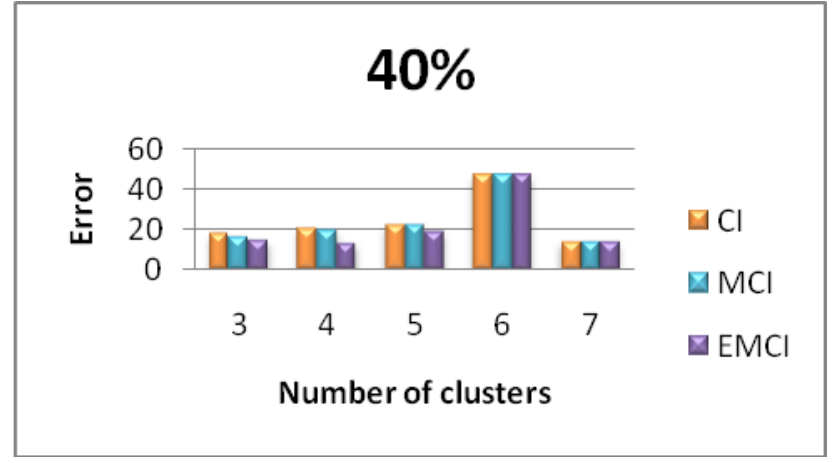

Fig. 2: A mean square error comparison of three imputation methods in the Bupa data set.

Table 3. A sum of square error comparison of three imputation methods in the Pima Indian data set.

\begin{tabular}{|c|c|c|c|c|}
\hline \multirow[t]{2}{*}{ Miss. $(\%)$} & \multirow[t]{2}{*}{ Cluster No } & \multicolumn{3}{|c|}{ Imputation approaches based K-mean } \\
\hline & & CI & MCI & EMCI \\
\hline \multirow{6}{*}{2} & 3 & 14.37554 & 13.25002 & 8.890231 \\
\hline & 4 & 13.85666 & 12.84985 & 6.06536 \\
\hline & 5 & 13.37925 & 12.84137 & 5.971876 \\
\hline & 6 & 11.82608 & 11.45384 & 5.877658 \\
\hline & 7 & 5.825113 & 5.825112 & 5.916673 \\
\hline & 8 & 7.825108 & 7.825106 & 7.825106 \\
\hline \multirow{6}{*}{4} & 3 & 18.03262 & 17.36147 & 13.55296 \\
\hline & 4 & 18.93389 & 17.38975 & 12.54147 \\
\hline & 5 & 18.04683 & 17.85205 & 12.71843 \\
\hline & 6 & 16.84882 & 16.74036 & 11.74633 \\
\hline & 7 & 11.36014 & 11.36014 & 11.91438 \\
\hline & 8 & 11.42276 & 11.42276 & 11.42276 \\
\hline \multirow{6}{*}{6} & 3 & 24.44564 & 23.20973 & 17.91235 \\
\hline & 4 & 22.63036 & 21.62001 & 16.1246 \\
\hline & 5 & 20.38086 & 20.01029 & 15.99459 \\
\hline & 6 & 20.81671 & 20.33147 & 14.35323 \\
\hline & 7 & 14.01545 & 14.01545 & 14.26166 \\
\hline & 8 & 14.28277 & 14.28277 & 14.28277 \\
\hline \multirow{6}{*}{10} & 3 & 38.09534 & 36.341 & 33.59278 \\
\hline & 4 & 33.94895 & 33.3278 & 29.87464 \\
\hline & 5 & 33.55535 & 33.02003 & 30.1552 \\
\hline & 6 & 32.1873 & 31.36938 & 27.59835 \\
\hline & 7 & 26.59483 & 26.59483 & 27.42991 \\
\hline & 8 & 26.49616 & 26.49616 & 26.49616 \\
\hline \multirow{6}{*}{20} & 3 & 64.64467 & 62.69846 & 64.57974 \\
\hline & 4 & 64.20623 & 63.53032 & 64.39197 \\
\hline & 5 & 81.72205 & 81.28112 & 70.34858 \\
\hline & 6 & 64.43491 & 63.68247 & 59.91234 \\
\hline & 7 & 57.20836 & 57.20828 & 58.84161 \\
\hline & 8 & 57.41436 & 57.41428 & 57.41428 \\
\hline
\end{tabular}

\begin{tabular}{|l|r|rrr|}
\hline \multirow{3}{*}{40} & 3 & 119.333 & 117.3868 & 127.27 \\
& 4 & 119.7724 & 119.106 & 127.9656 \\
& 5 & 118.6994 & 118.2354 & 127.2739 \\
& 6 & 120.2016 & 119.4625 & 117.9062 \\
& 7 & 113.2273 & 113.2272 & 115.7847 \\
& 8 & 145.5723 & 145.5722 & 145.5722 \\
\hline
\end{tabular}

Table 3 illustrates an error comparison between an imputation methods based on k-means, CI, MCI and EMCI in different missing instance percentage at several cluster number for Pima Indian dataset.

From table 3 we can notice that EMCI is still better than the other two methods. For higher level of missing 20 and 40 percentage for little clusters 3 and 4 the MCI is better, See figure 3 .
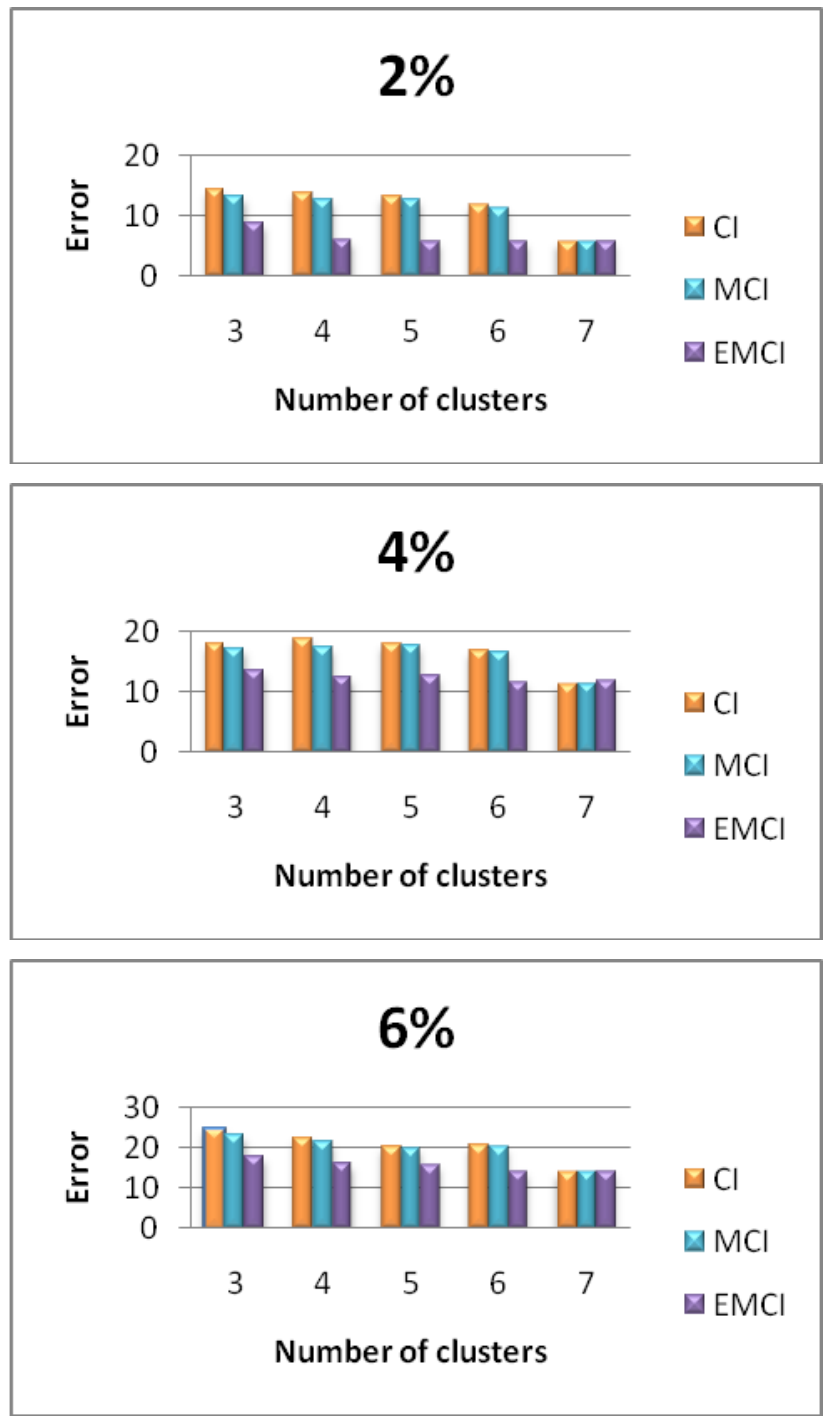

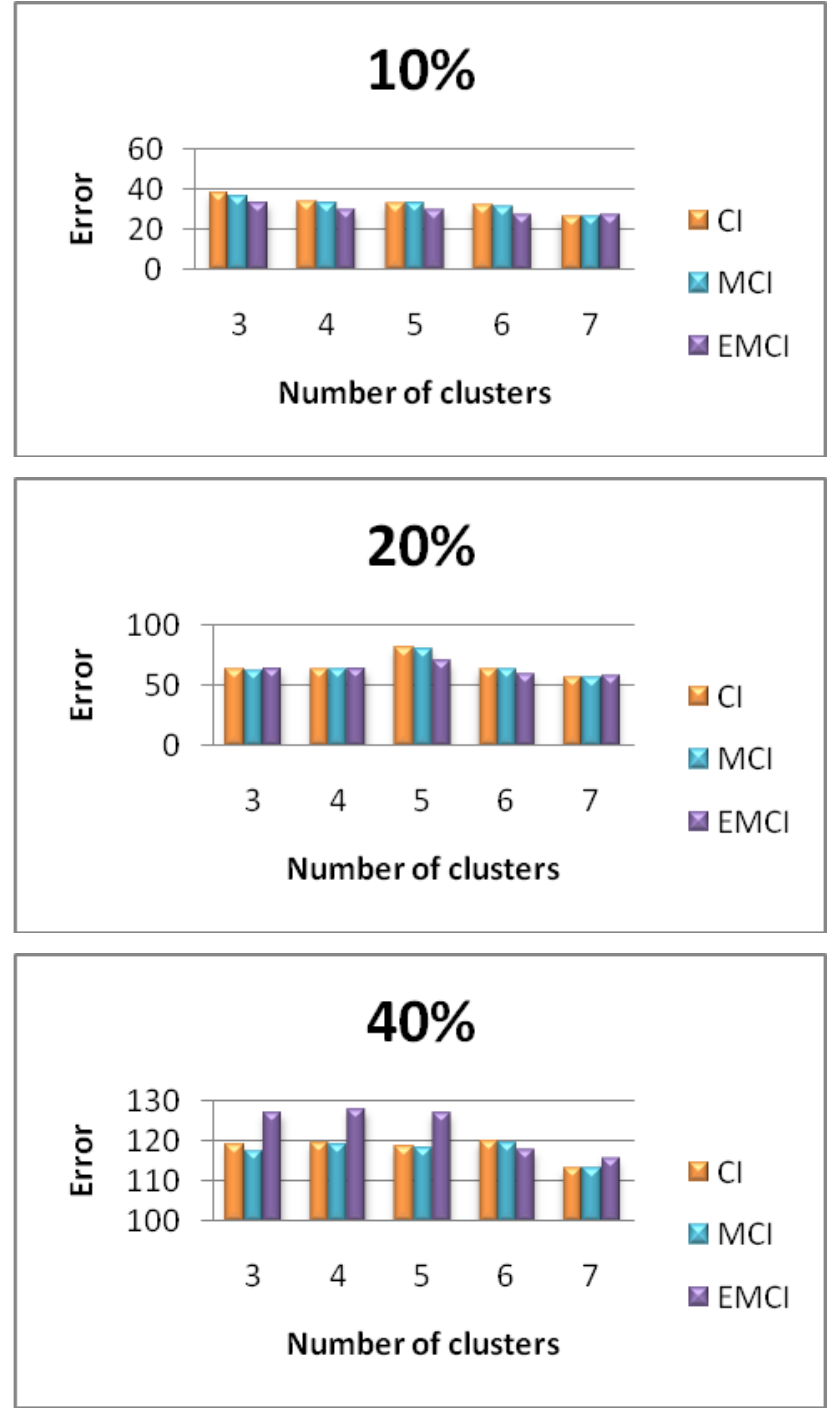

Fig. 3: A mean square error comparison of three imputation methods in the Pima Indian data set.

Table 4 illustrates an error comparison between an imputation methods based on k-means, CI, MCI and EMCI in different missing instance percentage at several cluster number for Ecoli data set.

Table 4. A sum of square error comparison of three imputation methods in the Ecoli data set.

\begin{tabular}{|r|r|rrr|}
\hline Miss.(\%) & \multicolumn{2}{|l|}{$\begin{array}{l}\text { Cluster } \\
\text { No }\end{array}$} & \multicolumn{3}{|c|}{ Imputation } & approaches based K-mean \\
& & CI & MCI & EMCI \\
\hline \multirow{3}{*}{2} & 4 & 5.41087 & 4.531235 & 4.075765 \\
& 5 & 9.091621 & 8.014148 & 3.787097 \\
& 5 & 4.477868 & 4.462628 & 3.313772 \\
& 6 & 2.875017 & 2.875017 & 3.065405 \\
& 7 & 2.676449 & 2.676449 & 2.676449 \\
\hline
\end{tabular}

\begin{tabular}{|r|r|rrr|}
\hline \multirow{3}{*}{4} & 3 & 6.562714 & 5.990744 & 6.461079 \\
& 4 & 10.32932 & 8.963055 & 6.190022 \\
& 5 & 6.512977 & 6.551205 & 5.492534 \\
& 6 & 4.635415 & 4.635415 & 5.314992 \\
& 7 & 4.161032 & 4.161032 & 4.161032 \\
\hline \multirow{6}{*}{6} & 3 & 7.599198 & 7.035617 & 9.206245 \\
& 4 & 11.0807 & 9.722828 & 8.954317 \\
& 5 & 7.268047 & 7.306274 & 7.490683 \\
& 6 & 5.395188 & 5.395188 & 5.761791 \\
& 7 & 5.368557 & 5.368557 & 5.368557 \\
\hline \multirow{6}{*}{20} & 3 & 9.096494 & 8.35237 & 10.79339 \\
& 4 & 12.58382 & 11.04685 & 9.157546 \\
& 5 & 10.30394 & 10.36206 & 8.858151 \\
& 6 & 6.638017 & 6.638016 & 7.07966 \\
& 7 & 6.767209 & 6.767208 & 6.767208 \\
\hline \multirow{6}{*}{$*$} & 3 & 14.13585 & 13.38966 & 22.07389 \\
& 4 & 17.63459 & 16.09575 & 19.19012 \\
& 6 & 16.69709 & 16.76461 & 18.83994 \\
& 7 & 15.41178 & 15.41178 & 15.41178 \\
\hline
\end{tabular}

From table 4 we can notice that the two methods MCI and EMCI is better than CI and, in many cases, the MCI is better than the EMCI but the EMCI is better in other cases, See figure 4 .
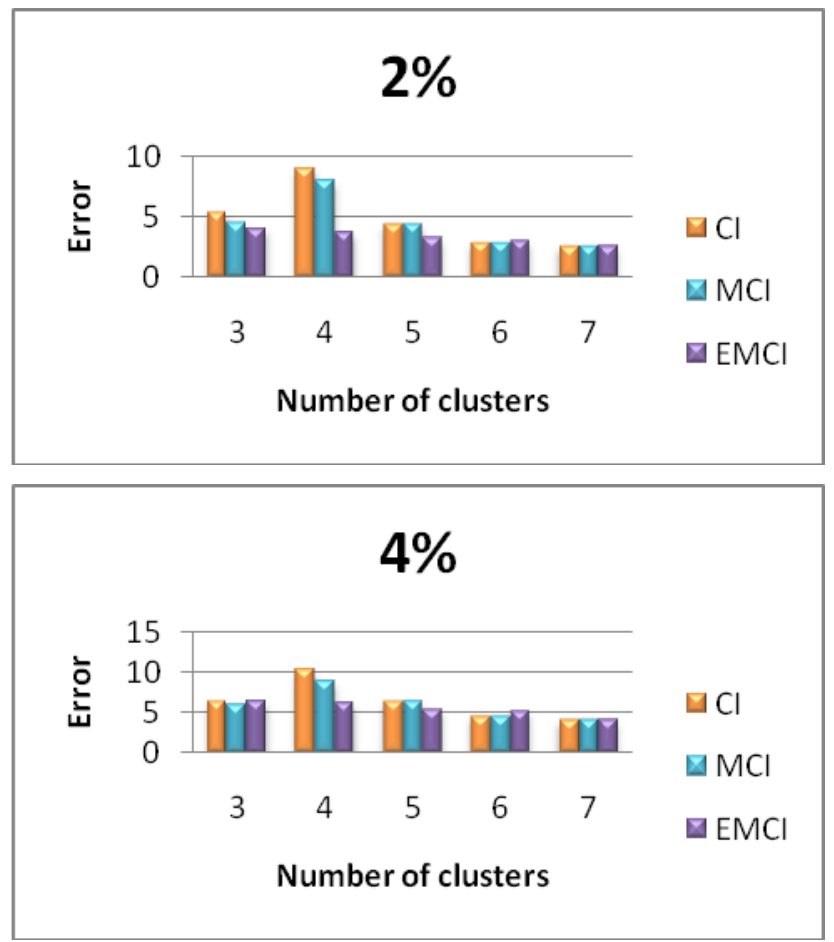

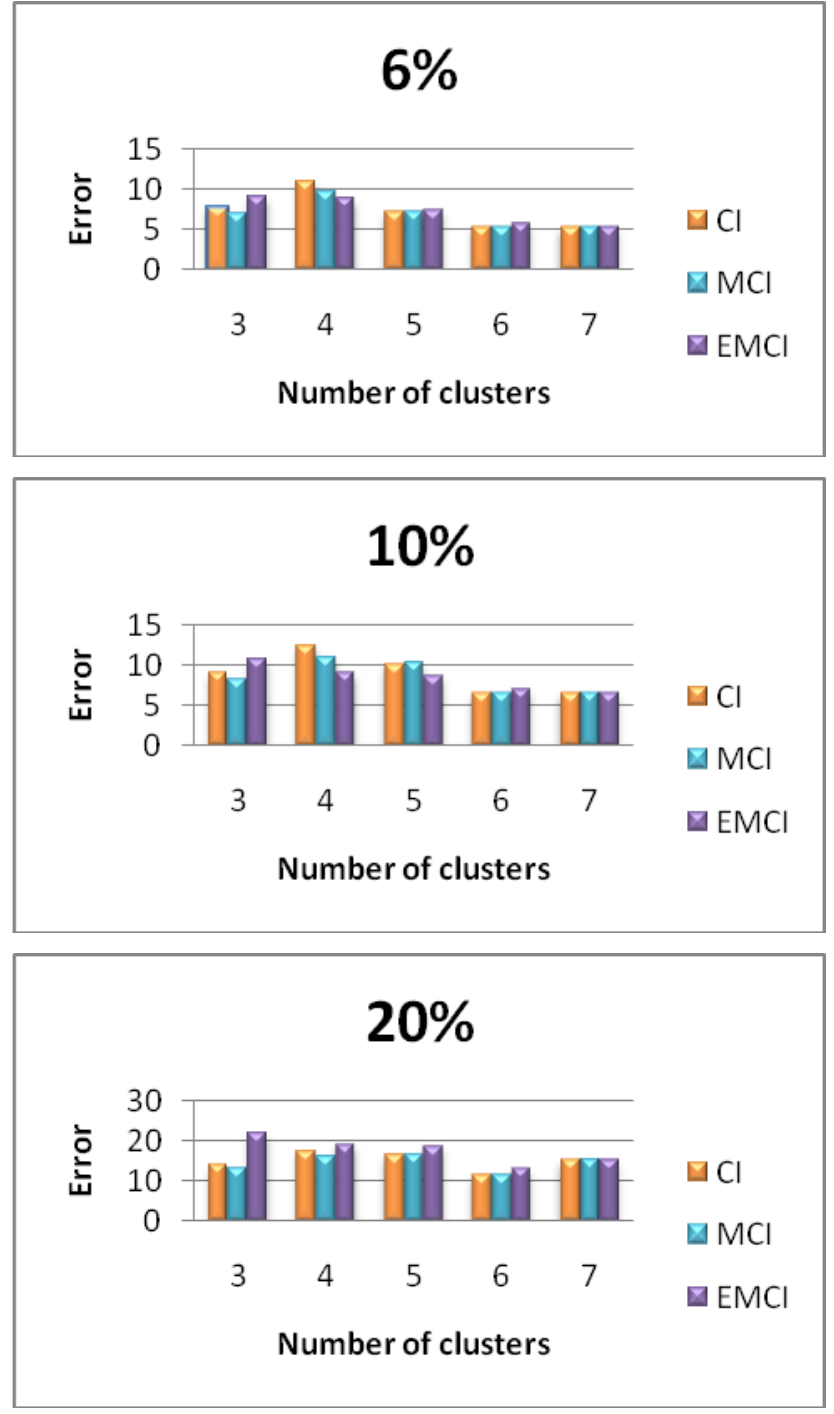

Fig. 4: A mean square error comparison of three imputation methods in the Ecoli data set.

Table 5 illustrates an error comparison between an imputation methods based on k-means, CI, MCI and EMCI in different missing instances of percentage at several cluster number for Iris data set.

Table 5. a sum of square error comparison of three imputation methods in the Iris data set.

\begin{tabular}{|r|r|rrr|}
\hline Miss.(\%) & $\begin{array}{l}\text { Cluster } \\
\text { No }\end{array}$ & Imputation & approaches based K-mean \\
& & CI & MCI & EMCI \\
\hline \multirow{3}{*}{2} & 3 & 0.447209 & 0.447209 & 0.447209 \\
& 4 & 0.420188 & 0.420188 & 0.420188 \\
& 5 & 0.420188 & 0.420188 & 0.420188 \\
& 6 & 0.457572 & 0.457572 & 0.457572 \\
& 7 & 0.536267 & 0.536267 & 0.536267 \\
\hline
\end{tabular}

\begin{tabular}{|c|c|c|c|c|}
\hline \multirow{5}{*}{4} & 3 & 1.018319 & 1.018319 & 1.06553 \\
\hline & 4 & 0.790119 & 0.79012 & 0.79012 \\
\hline & 5 & 0.790119 & 0.79012 & 0.79012 \\
\hline & 6 & 1.105146 & 1.105146 & 1.105146 \\
\hline & 7 & 1.278171 & 1.278171 & 1.278171 \\
\hline \multirow{5}{*}{6} & 3 & 1.438984 & 1.438984 & 0.893171 \\
\hline & 4 & 1.066982 & 1.066982 & 1.066982 \\
\hline & 5 & 1.066982 & 1.066982 & 1.066982 \\
\hline & 6 & 1.588675 & 1.588675 & 1.588675 \\
\hline & 7 & 1.926627 & 1.926627 & 1.926627 \\
\hline \multirow{5}{*}{10} & 3 & 2.252538 & 2.252538 & 1.755318 \\
\hline & 4 & 1.629589 & 1.629589 & 1.629589 \\
\hline & 5 & 1.629589 & 1.629589 & 1.629589 \\
\hline & 6 & 2.499124 & 2.499124 & 2.499124 \\
\hline & 7 & 3.06347 & 3.063469 & 3.063469 \\
\hline \multirow{5}{*}{20} & 3 & 4.289041 & 4.28904 & 4.832908 \\
\hline & 4 & 3.045854 & 3.045854 & 3.045854 \\
\hline & 5 & 3.045854 & 3.045854 & 3.045854 \\
\hline & 6 & 4.535299 & 4.535299 & 4.535299 \\
\hline & 7 & 5.684607 & 5.684606 & 5.684606 \\
\hline \multirow{5}{*}{40} & 3 & 6.942563 & 6.942573 & 7.727729 \\
\hline & 4 & 9.3234 & 9.323404 & 9.323404 \\
\hline & 5 & 4.254536 & 4.254535 & 4.254535 \\
\hline & 6 & 6.270786 & 6.270789 & 6.270789 \\
\hline & 7 & 7.323775 & 7.323776 & 7.323776 \\
\hline
\end{tabular}

From table 5 we can notice that the three algorithms are same in most cases. EMCI is better for level of missing value 6 and 10 percentage for little clusters as 3, See figure 5 .

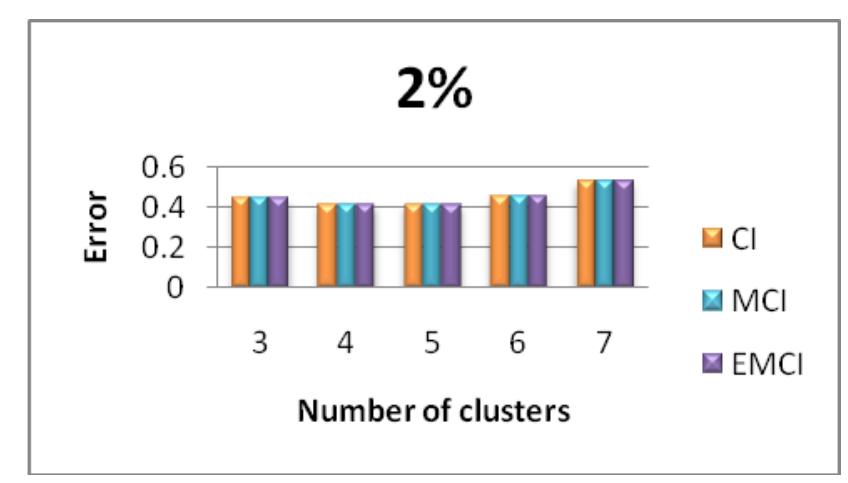



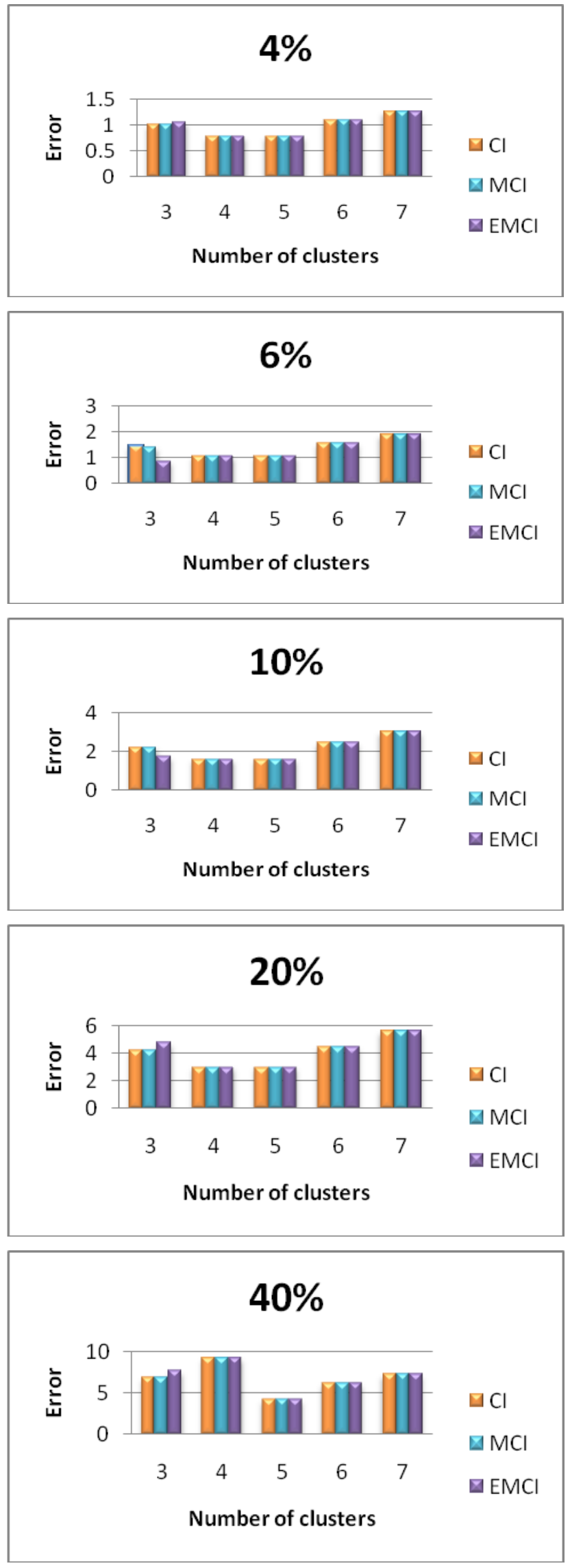

Fig. 5: A mean square error comparison of three imputation methods in the Iris data set.

\section{CONCLUSIONS}

Missing data is a usual drawback in many real-world applications. A classical solution is imputation i.e., to estimate and to fill in the unknown values using available data. This work analyzes the behavior of three imputation methods based on k-means; a classic imputation (CI), a modification of classic imputation (MCI) and enhancement of modification of classic imputation (EMCI). The first method (CI) is used and gives higher accuracy than Mean, Mode, Median and c4.5 on dataset such as Bupa, Pima Indian, and e.t. Our proposed methods; (MCI) and (EMCI) is better than the classic (CI). In most cases when the number of clusters is less, the performance of EMCI is better than the two others methods and MCI is better than CI. When the number of clusters is increased the three algorithms are the same.

\section{REFERENCES}

[1] Jiawei, H. and Micheline, K., 2006. Data mining Concept and Techniques. 2nd Edn Morgon Kaufmaan Publishers. ISBN: 1-55860-901-6.

[2] Mehala, B., Vivekanandan K. and Ranjit Jeba Thangaiah, P., 2008. An Analysis on K-Means Algorithm as an Imputation Method to Deal with Missing Values. Asian Journal of Information Technology 7 (9): 434-441.

[3] Lakshminarayan, K., Harp, S. A. and Samad, T., 1999. Imputation of missing data in industrial database, Apple. Intell. 11, 259-275.

[4] Jau-Huei Lin and Peter J. Haug, 2008. Exploiting missing clini- cal data in Bayesian network modeling for predicting medical problems Journal of Biomedical Informatics 41, 1-4.

[5] Alireza farhangfar, Lukase Kurgan and Jennifer Dy, 2008. Impact of imputation of missing values on classification error for discrete data. Pattern Recognition $41,3692-3705$.

[6] Dempster, A.P. and LairdandDB Rubin, R. J., 1977. Maximum likelyhood from incomplete data via the EM algoritm (with Discussion). I. R. Stat. Soc, B39: 1-38. http://wwwjstororg/pss/2984875.

[7] Daqian, G. and Yang, G. 2005. Incremental gradent descent imputation method for missing data in learning classifier systems. GECCO, ACM, Wash- ington, DC, USA, pp: 72-73.

[8] Fulufhelo, V., Nelwamondo and Tshlidzi, M. 2007. Rough sets computations to impute missing data. Comput. Vision and Pattern Recog., 1, 1-19.

[9] Musil, C.M., Wamer, C.B., Yobas, P.K. and Jones, S.L. 2002. A comparison of imputation techniques for handling missing data. Western J. Nus. Res., 24 (5).

[10] Cristian P., D., Alain, P. Monique and Tahar, K. 2005. Tools for statistical analysis with missing data: Appli cation to a large medxal database. ENMI, pp: 181-186.

[11] Joseph L. Schafer and Maren K. Olsen, 1998. Multiple Imputation for multivariate Missing data problems: a data analyst's perspective, 33, 545--571.

[12] Pedro J.Garc Laencina, Jose'-Luis Sancho-Gomez, Anbal R.Figueiras-Vidal and Michel Verleysen, 2009. K nearest neighbours with mutual information for simultaneous 
Classification and missing data imputation. Neurocomputing 72, 1483-1493.

[13] Allison, P. D., 2001. Missing data, Sage University Papers Serieson Quantitative Applications in the Social Sciences, Thousand Oaks, California, USA.
[14] Little, R. J. A. and Rubin, D. B. Statistical 2002. Analysis with Missing Data, seconded, Wiley, NJ, USA.

[15] Sande, G. 1983. Hot Deck Imputation Procedures, Incomplete data in Sample Surveys, vol. 3, Academic Press. 